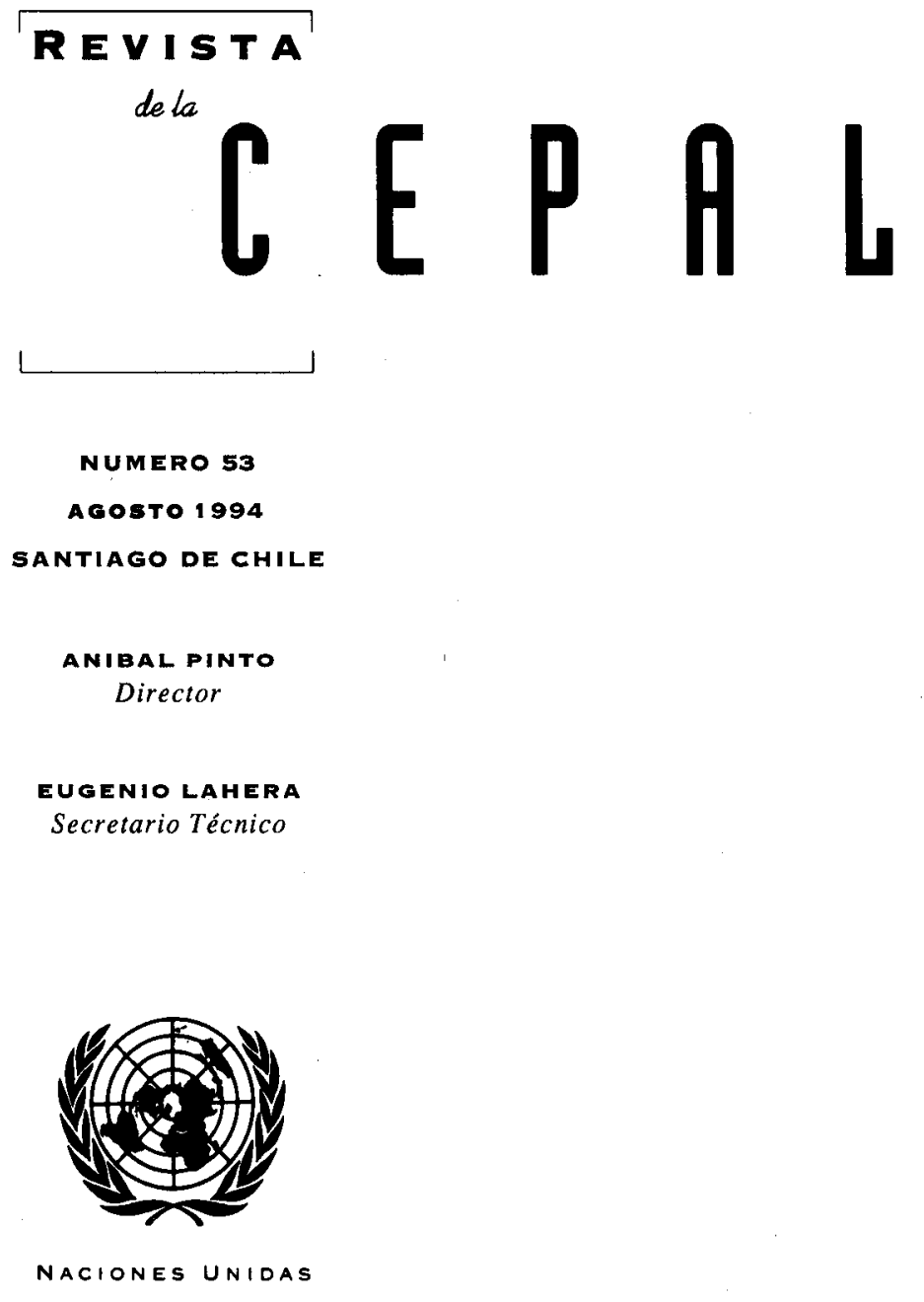


América Latina y el Caribe frente a la economía mundial

Gert Rosenthal

Afluencia de capitales externos y políticas macroeconómicas

Andras Uthoff y Daniel Titelman

Represión fínanciera y patrón de financiamlento latínoamericano

31

Marcos Antonio Macedo Cintra

Políticas de competitividad

49

Wilson Peres

Política industrial y fomento de la competitividad

Osvaldo Rosales

El regionalismo abierto y la integración económica

Juan A. Fuentes $K$.

Transformaciones del trabajo femenino urbano

91

Irma Arriagada

La gestión del agua y las cuencas en América Latina

Axel Dourojeanni

Políticas públicas y competitividad de las exportaciones

agrícolas

Milton von Hesse

Agroindustria y transformación productiva de la pequeña agricultura

Alejandro Schejtman

Grupos privados nacionales en México, 1988-1993

Celso Garrido

Evolución y perspectivas de la reforma y la apertura en China 


\section{La gestión del agua $y$ las cuencas en América Latina}

\section{Axel Dourojeanni}

Director de la

División de Recursos

Naturales y Energía

de la CEPAL.
La sustentabilidad o sostenibilidad del desarrollo es un concepto abstracto si no se asocia a objetivos claros que se deben alcanzar dentro de territorios definidos, y a los procesos de gestión necesarios para hacerlo. La gestión de los recursos naturales dentro del territorio de una cuenca hidrográfica es una opción valiosa para guiar y coordinar procesos de gestión para el desarrollo, considerando las variables ambientales. Para convertir las políticas ambientales en acciones es preciso disponer de entidades adecuadas de gestión, normalmente muy complejas. La creación de estas entidades significa generar un sistema público-privado autónomo en lo financiero, orientado a lo social y sensible a los aspectos ambientales, que además debe actuar en forma democrática y participativa. La creación de entidades para orientar la gestión de los recursos naturales de una cuenca, sobre todo del agua, ha despertado el interés de los países de la región, con diversos resultados. Este interés se ha convertido hoy en una necesidad imperiosa, en vista de la mayor competencia por el uso múltiple del agua, y la necesidad de controlar la contaminación y de manejar el medio ambiente. El presente artículo considera algunos de los elementos esenciales que deben tomarse en cuenta al proponer la creación de tales entidades, aporta conceptos sobre gestión a nivel de cuencas y definiciones sobre los diversos enfoques con que se aborda el tema, y ofrece algunas recomendaciones para mejorar la formulación de las políticas y el funcionamiento de los sistemas de gestión integral del agua y de las cuencas. 


\section{El desarrollo sustentable y las}

\section{cuencas hidrográficas}

Una de las mayores preocupaciones actuales, por lo menos en las declaraciones de política, es la de encontrar opciones viables de desarrollo basadas en un crecimiento económico y equitativo, y que perdure en el tiempo. Esto último se ha revalorizado últimamente, al percatarse el hombre que muchos de sus llamados avances, sobre todo en materia de transformación productiva, se encuentran descompensados por los daños que ocasionan al ambiente.

La mayor conciencia y comprensión que hay actualmente de la interacción del hombre con el medio ambiente, y de la vulnerabilidad del desarrollo que no la toma en cuenta, se han hecho explícitas al agregarse al vocablo desarrollo el término sustentable o sostenible. Dado que la sustentabilidad debe estar implícita en el concepto de desarrollo, la palabra sustentable o sostenible debería ser sólo un agregado transitorio, válido únicamente hasta que la necesidad de duración en el tiempo se internalice definitivamente en él.

Por otro lado, la sustentabilidad del desarrollo sólo es una idea o una aspiración abstracta si el concepto no se asocia tanto a objetivos claros para alcanzar dentro de un territorio definido -que contiene los elementos y recursos naturales necesarios para la subsistencia de la especie humana- como a los procesos de gestión para alcanzar dichos objetivos. Las políticas de intención (politics) deberán transformarse en políticas de ejecución (policies), y es precisamente aquí donde surgen los mayores desafíos. 1

En la región se han denunciado ampliamente los problemas ambientales, se ha teorizado sobre la temática ambiental, se han promulgado leyes y hasta se han creado algunos ministerios del medio ambiente. Lo que sin embargo no se ha construido en forma suficiente son las bases para manejar cada uno de los recursos naturales _-el agua, los suelos, los bosques,

\footnotetext{
1 A lo largo de este trabajo se hace uso deliberado de algunas expresiones en inglés con el objetivo de afinar y precisar la tecnología sobre el tema, que suele pecar de ambigua en su relación entre español e inglés.
}

la fauna, los minerales y la energía - o algunos territorios naturales como las franjas costeras, las cuencas hidrográficas y los desiertos.

Esto indica que se han planteado metas muy amplias sin determinar los pasos necesarios para alcanzarlas. La organización de territorios para el manejo de cada recurso y luego del ambiente en general; la organización y capacitación de la población; la investigación de los ecosistemas; el establecimiento de los sistemas de gestión por territorio; el refuerzo de las instituciones públicas, sobre todo de los municipios, para apoyar la gestión ambiental; la valorización económica de recursos naturales; el mantenimiento de cuentas de patrimonio natural, y la elaboración de manuales y normas de trabajo, son aspectos necesarios para hacer avances concretos en el manejo de los recursos naturales y del entorno en general.

El manejo de los recursos naturales en el marco de la dinámica de una cuenca hidrográfica, más conocido como gestión de cuencas, surge como una de las posibles opciones para articular la participación de los usuarios de recursos naturales en materia de gestión ambiental. La cuenca hidrográfica posee un valor único como base para coordinar actores ligados a un recurso común, el agua, y para evaluar los efectos que tienen sobre este recurso las medidas de gestión ambiental. La calidad del agua refleja en gran parte la capacidad de gestión ambiental dentro de la cuenca.

Una primera aproximación a la gestión de cuencas es la de limitarla a la gestión de los recursos hídricos presentes en dicho territorio. La gestión del agua es un proceso complejo destinado a controlar el ciclo de un recurso natural cuya presencia es errática e irregular en el tiempo y en la superficie terrestre. Además, el agua es vulnerable al trato que se le dé, ya que se puede contaminar fácilmente, alterándose así todos sus usos posteriores, actuales o potenciales.

A través de este proceso se busca solucionar conflictos entre múltiples usuarios que, queriéndolo o no, dependen de un recurso compartido. Por ello, 
aunque pueden contar con concesiones o derechos de uso, no dejan de afectarse mutuamente y de ser interdependientes. La oferta proviene, usualmente, de un sistema común, y a él se vuelven a integrar los excedentes de uso y los efluentes. Las aguas superficiales, subterráneas y atmosféricas, así como las zonas de evacuación, forman de este modo una sola unidad.

Las acciones que se realicen tienen enormes repercusiones sobre la salud humana, el medio ambiente y la producción, por lo que deben ser abordadas en forma altamente técnica. El elevado costo de las obras, así como el largo tiempo de madura- ción de los proyectos hidráulicos, hacen aún más necesario que el sistema de gestión esté a cargo de expertos cuya permanencia no dependa de los cambios políticos.

Por último, el proceso de gestión del agua requiere que muchos agentes actúen en forma coordinada, no obstante su diversidad de criterios y el hecho de que algunos de ellos desconocen los efectos de sus decisiones sobre el ciclo hidrológico. De ahí la importancia de disponer de mecanismos estables de coordinación y, por lo menos, de un centro o autoridad de cuenca permanente.

\section{II}

\section{Las características de las aguas y las cuencas}

La cuenca hidrográfica es un territorio que es delimitado por la propia naturaleza, ${ }^{2}$ esencialmente por los límites de las zonas de escurrimiento de las aguas superficiales que convergen hacia un mismo cauce. La cuenca, sus recursos naturales y sus habitantes poseen condiciones físicas, biológicas, económicas, sociales y culturales que les confieren características peculiares.

Físicamente, representa una fuente natural de captación y concentración de agua superficial y subterránea $\mathrm{y}$, por lo tanto, tiene una connotación esencialmente volumétrica e hidrológica. Al mismo tiempo, tanto la cuenca como, sobre todo, el agua captada por ella, es una fuente de vida para el hombre. Pero también es origen de riesgo cuando ocurren fenómenos naturales extremos o se producen alteraciones por contaminación.

En zonas de altas montañas y cordilleras las cuencas son ejes naturales de comunicación y. de integración comercial, sea a lo largo de sus ríos, sea a lo largo de las cumbres que las separan. Es decir, hay estrechos mecanismos de interacción de sus habitantes que les confieren condiciones socioeconómicas particulares.

En cuencas con grandes descargas de agua y amplios valles relativamente planos, el eje de los ríos se

\footnotetext{
${ }^{2}$ Guillermo Cano y Joaquin López han dicho que "Dios estableció las líneas del divorcio de las aguas como límites naturales de las cuencas hídricas. Los hombres, para sus menesteres políticos y administrativos, han trazado otras, que generalmente se entrecruzan y no coinciden con aquéllas" (Cano y López, 1976).
}

constituye también en una zona de articulación de sus habitantes, sobre todo por el uso del cauce del río para navegación, transporte y comunicación.

El territorio de las cuencas facilita la relación entre quienes viven en ellas, aunque se agrupen en comunas por razones político-administrativas, debido a su dependencia común de un sistema hídrico compartido y de los caminos y vías de acceso, y al hecho de que deben enfrentar peligros comunes. Cuando no existen sistemas de conciliación de intereses entre los diferentes actores que dependen de una cuenca se producen conflictos entre ellos.

Esto se observa ciertamente en cuencas habitadas que se ubican en cordilleras con altas montañas (watersheds), pero también se da en amplias cuencas fluviales (river basins) con trabajos hidráulicos que benefician a los habitantes en su conjunto y que por lo tanto les crean una dependencia común. ${ }^{3}$

En las cuencas hidrográficas es fácil percibir el efecto negativo de las acciones del hombre sobre su entorno, sobre todo por la contaminación del agua. Esto se plantea, por ejemplo, en las bases de la creación de agencias de cuencas en Francia: se dice allí que el medio acuático es una entidad que alberga y sostiene todo un mundo animal y vegetal, y que sus aguas y sus riberas conforman una construcción bio-

\footnotetext{
${ }^{3}$ En el idioma español no existe la distinción entre watershed y river basin. Se traducen ambos conceptos como cuenca hidrográfica, aunque se procura hacer distinciones con expresiones tales como cuenca fluvial y hoya hidrográfica para referirse a river basin, y cuenca de alta montaña o cuenca de captación para referirse a watershed.
} 
lógica particular. La intervención impensada del hombre sobre uno solo de estos elementos rompe este equilibrio precario y empobrece el medio natural. Por lo tanto, para una gestión armónica de los recursos hídricos es necesario: i) ante todo, reconocer que una cuenca hidrológica o hidrogeológica constituye una unidad; ii) considerar y preservar esta unidad es condición esencial para dar satisfacción óptima a la demanda de agua de los diferentes usuarios; iii) definir objetivos específicos y apropiados a cada ámbito o territorio, y ejecutar las obras y acciones requeridas para alcanzar tales objetivos; iv) aceptar que todos los usuarios tienen un legítimo derecho al agua y que, en consecuencia, cada uno de ellos tiene en forma equivalente limitaciones para su uso propio.

La cuenca es una unidad natural que se presta bien como territorio para articular los procesos de gestión que tienden eventualmente al desarrollo sostenible. A su vez, los procesos de gestión del agua tienen su propia complejidad

\section{III}

\section{La cuenca como opción territorial para dirigir procesos de gestión ambiental}

El territorio que abarca una cuenca hidrográfica no es ciertamente el único ámbito dentro del cual se pueden dirigir y coordinar acciones de desarrollo de modo de considerar la temática ambiental. Los límites de las aguas superficiales, que forman la cuenca, no necesariamente coinciden con los límites de las aguas subterráneas, obviamente no abarcan las superficies de los mares donde se genera una gran parte del ciclo hidrológico, y son menos relevantes en zonas relativamente planas y de extrema aridez.

El uso del territorio de una cuenca para fines de gestión ambiental, por lo tanto, es sólo una opción con mayor o menor validez, según las características geográficas de su entorno. Es una opción importante desde el punto de vista ambiental porque, tal como se mencionó, propicia la coordinación entre usuarios unidos a un mismo recurso, como el agua, y sobre todo facilita la verificación de los progresos en el control de la contaminación a través de sus efectos en la calidad del agua. Esto, sin embargo, no hace que el territorio de una cuenca sea el único espacio requerido para la gestión de los recursos naturales o del ambiente en general.

Esta observación es importante para desmitificar la creencia de algunas personas de que se puede dirigir todo el desarrollo de una región o el manejo ambiental exclusivamente a partir de demarcaciones dadas por límites de cuencas. Puede decirse que tener en cuenta los límites de las cuencas es una condición necesaria para considerar aspectos ambientales, sobre todo del agua y de sus recursos "asociados", pero no es suficiente como jurisdicción para gestionar el desarrollo del hombre.

En este sentido es fundamental que toda propuesta de gestión a nivel de cuenca se haga teniendo en cuenta su relación con los sistemas de gestión que funcionan con otros límites, sobre todo con las demarcaciones político-administrativas, entre las cuales los municipios son prioritarios. Ha de quedar claro que para llevar a cabo los procesos de gestión de cuencas es preciso coordinar la acción de las variadas autoridades públicas y privadas que actúan sọbre el territorio de la cuenca.

Por ejemplo, los trabajos de manejo de cuencas que toman en cuenta a los municipios, como los realizados con la participación de 74 de ellos en la cuenca del río Chicamocha en Colombia (Acero Suárez, 1993, pp. 13 a 17), tienen mucho más probabilidades de éxito si las alcaldías tienen responsabilidades en la ejecución de algunas acciones del proyecto. A la inversa, un programa municipal que pretende mejorar el ambiente o prevenir efectos negativos en el entorno debe considerar la influencia de las cuencas hidrográficas que se relacionan total o parcialmente con su jurisdicción.

A escalas de cuencas mayores, la misma relación debe darse entre las autoridades de límites político-administrativos y de límites naturales. Por ejemplo, la jefatura de un proyecto de desarrollo o de la gestión integral de una gran cuenca debe coordinarse con las autoridades de desarrollo de la región donde se encuentra ubicada la cuenca. En muchos casos ha ocurrido que, a falta de tal coordinación, 
una de las dos autoridades (la de desarrollo de cuencas o la de desarrollo regional) ha absorbido a la otra, o bien ambas han coexistido en conflicto permanente.

\section{IV}

\section{Definiciones y alcances de los procesos de gestión de cuencas}

El tema de la gestión de cuencas ha evolucionado mucho en la región, pero a pesar de los avances logrados aún no hay consenso sobre definiciones que precisen los objetivos de dicha gestión. La falta de claridad conceptual en la materia atenta todavía contra el intercambio de información sobre sus experiencias entre los países, crea cruces de funciones $y$, sobre todo, dificulta la formulación de políticas y leyes sobre el tema.

El cuadro 1 sintetiza y ordena los conceptos y la terminología con que se enfoca el tema de la gestión de cuencas hidrográficas en América Latina y el Caribe. Se ha construido como una matriz que relaciona las etapas del proceso de gestión de cuencas con los objetivos de dicho proceso, definidos por la cantidad de elementos y recursos que abarca la gestión. Se busca así facilitar la comprensión de las acciones que se pueden coordinar en una cuenca, y de los fines perseguidos con dicha coordinación. Se ha considerado útil además dilucidar algunas complejidades adicionales planteadas por ciertas discrepancias terminológicas entre el inglés y el español, por lo cual en el cuadro se incluyen algunas denominaciones en ambos idiomas, que pueden facilitar la comprensión de los términos en español al relacionarlos con el concepto que les dio origen. Esta forma de analizar los términos es inédita y se espera que ayude a clasificar concepciones sobre los objetivos de la gestión de cuencas.

CUADRO 1

Gestión en cuencas: etapas y objetivos

\begin{tabular}{|c|c|c|c|}
\hline \multicolumn{4}{|c|}{ Objetivos de la gestión } \\
\hline $\begin{array}{l}\text { Etapas } \\
\text { de } \\
\text { gestión }\end{array}$ & $\begin{array}{l}\text { Aprovechamiento y } \\
\text { manejo de los } \\
\text { recursos hídricos de } \\
\text { la cuenca }\end{array}$ & $\begin{array}{l}\text { Aprovechamiento y manejo de } \\
\text { todos los elementos y } \\
\text { recursos naturales en la } \\
\text { cuenca }\end{array}$ & $\begin{array}{l}\text { Aprovechamiento y manejo } \\
\text { integrado de todos los } \\
\text { elementos y recursos y de la } \\
\text { infraestructura construida } \\
\text { por el hombre para } \\
\text { habilitar la cuenca }\end{array}$ \\
\hline $\begin{array}{l}\text { Etapa } \\
\text { inicial }\end{array}$ & $\begin{array}{l}\text { Estudios y formulación } \\
\text { de planes y proyectos }\end{array}$ & $\begin{array}{l}\text { Estudios y formulación } \\
\text { de planes y proyectos }\end{array}$ & $\begin{array}{l}\text { Estudios y formulación } \\
\text { de planes y proyectos }\end{array}$ \\
\hline 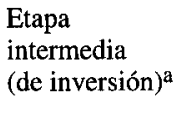 & $\begin{array}{l}\text { Desarrollo o aprovechamiento } \\
\text { de recursos hídricos } \\
\text { (Water resources } \\
\text { development) }\end{array}$ & $\begin{array}{l}\text { Desarrollo o aprovechamiento } \\
\text { de recursos naturales } \\
\text { (Natural resources } \\
\text { development) }\end{array}$ & $\begin{array}{l}\text { Desarrollo de } \\
\text { cuencas } \\
\text { (River basin } \\
\text { development) }\end{array}$ \\
\hline $\begin{array}{l}\text { Etapa } \\
\text { permanente } \\
\text { (de operación) }^{b}\end{array}$ & $\begin{array}{l}\text { Administración de } \\
\text { recursos hídricos } \\
\text { (Water resources } \\
\text { management) }\end{array}$ & $\begin{array}{l}\text { Manejo de } \\
\text { recursos naturales } \\
\text { (Natural resources } \\
\text { management) }\end{array}$ & $\begin{array}{l}\text { Gestión ambiental } \\
\text { (Environmental } \\
\text { management) }\end{array}$ \\
\hline \multicolumn{4}{|c|}{$\begin{array}{l}\text { Manejo de cuencas } \\
\text { (Watershed management) }\end{array}$} \\
\hline
\end{tabular}

Fuente: Elaboración del autor.

a Inversión para habilitar la cuenca a fin de aprovechar y manejar sus recursos naturales con miras al desarrollo humano.

b Operación y mantenimiento de las obras construidas y manejo y conservación de los recursos y elementos naturales. 
Las entidades que se encargan de cada una de las acciones de gestión de cuencas mencionadas pueden optar por llamarse corporaciones, empresas, agencias, comisiones, autoridades, programas o proyectos, direcciones, organismos, juntas o asociaciones. La le- gislación de cada país fija en general las funciones y atribuciones legales de cada una de estas modalidades de organización, debiéndosele agregar las específicas de los procesos de gestión de cuencas y los medios adecuados para la participación pública y privada.

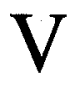

\section{La evolución de los sistemas de} gestión de cuencas

El tema de la gestión de cuencas se ha asociado históricamente con las principales culturas y civilizaciones que evolucionaron, y a veces desaparecieron, dependientes de la disponibilidad de agua. El hombre urbano, que constituye la mayoría de la población en muchos países, fue perdiendo la percepción de esa dependencia del agua, incluidos los cauces mismos, llegando al punto de ignorarla completamente a fuerza de tenerla en forma casi permanente. Se ha acostumbrado además a exigir que se aumente la oferta de agua en lugar de proponerse reducir su consumo con un uso más apropiado. Tampoco comprende a cabalidad que el agua es un recurso escaso, cuya presencia fluctúa en el tiempo y cuyo control exige grandes inversiones que deben planificarse con años de anticipación.

Cada cierto tiempo, sin embargo, alguna inundación, una sequía prolongada o la contaminación visible del agua le recuerda esa dependencia. Pero el efecto no siempre dura lo suficiente como para que se organice y actúe para equilibrar mejor la oferta y la demanda de agua, con visión de largo plazo, y sobre todo para establecer sistemas de gestión estables y con financiamiento garantizado.

Debido en parte a estas fluctuaciones en la percepción del valor del agua, en el presente siglo el tema de la gestión para la coordinación de acciones en una cuenca con propósitos múltiples, en América Latina, ha tenido una evolución que no ha sido ni uniforme ni estable. Los sistemas de gestión han venido cambiando cada vez más y en forma irregular; en varios casos, por lo menos en lo que toca al agua, la organización tendía a ser mejor en el pasado que ahora.

En sus etapas iniciales la coordinación de actividades a nivel de cuencas era limitada. Se hacían trabajos que tomaban en cuenta la cuenca hidrográ- fica para dar respuesta a problemas coyunturales o demandas específicas o sectoriales de agua: garantizar la navegación y mejorarla, abastecer de agua a poblaciones y zonas de riego, controlar inundaciones, mitigar sequías y construir centrales hidroeléctricas.

El siguiente paso consistió en operar y mantener las obras construidas. Esta gestión se limitaba a ocuparse de los sistemas construidos, sin mayor interés por el uso múltiple del agua ni por "manejar la cuenca" (es decir, por manejar los recursos naturales de la cuenca). Así, en la región surgieron diversos sistemas de gestión del agua por cuencas, gran parte de los cuales sólo se preocuparon del uso sectorial que se daba al agua, como en el caso del riego (por ejemplo, a través de Juntas de Vigilancia, en Chile, y Administraciones Técnicas de Distritos de Riego, en el Perú).

A partir de 1940 se crearon comisiones (México) y corporaciones para el desarrollo integral de cuencas (es decir, para el desarrollo regional a nivel de cuencas). Estas corporaciones se sustentaron en la construcción de obras hidráulicas para abarcar extensos territorios bajo su jurisdicción y realizar inversiones en múltiples sectores.

En épocas mucho más recientes (1970) apareció el "manejo de cuencas", principalmente con el fin de mitigar el aporte de sedimentos a los embalses construidos y controlar deslizamientos o inundaciones. Son pocos los casos en que se manejan todos los recursos naturales de la cuenca - flora, fauna, bosques y tierras- para aprovecharlos y conservarlos. Los proyectos agrosilvopastoriles ayudaron a avanzar en este sentido, pero no suplieron la carencia de un sistema de coordinación para el manejo de los recursos naturales por cuencas.

El tema ambiental surgió más tarde en Améri- 
ca Latina (unos cinco a siete años después de la reunión de Estocolmo en 1972). Primero hubo estudios de impacto ambiental y luego análisis ambientales. En gran medida, la gestión ambiental a nivel de cuencas no ha pasado de los estudios, y si no se manejan los recursos naturales en forma coordinada, ni siquiera el agua, será imposible hacer "gestión ambiental".

En resumen, la coordinación de acciones a nivel de cuencas en América Latina ha seguido el siguiente orden cronológico:

i) se enfrenta el tema del control y aprovechamiento del agua en cuencas con la construcción de obras hidráulicas (water resources development);

ii) se aborda la administración del agua en cuencas (water resources management);

iii) se pasa directamente al desarrollo de cuencas (river basin development);

iv) se considera el manejo de las cuencas de captación (watershed management), sobre todo para controlar la erosión que afecta a los embalses construidos, evitar deslizamientos y controlar torrentes;

v) se pasa directamente a tratar el tema de la gestión ambiental.

Lo que destaca en esta evolución es que se haya pasado abruptamente a coordinar, por lo menos en el papel, la gestión ambiental a nivel de cuenca y región ${ }^{4}$ sin haber todavía coordinado plenamente las medidas de desarrollo y gestión de por lo menos algunos de los principales recursos naturales de una cuenca, como el agua.

Actualmente el tema de las cuencas ha recobrado vigencia. Se espera que la clasificación y la revisión histórica de esta materia contribuya a que se propongan entidades adecuadas para realizar la gestión en cada caso, rescatando las experiencias valiosas del pasado.

En los años noventa la conjugación de una mayor preocupación ambiental con los efectos cada vez mayores de las catástrofes naturales (inundaciones, por ejemplo) en las obras construidas por el hombre, y la aparición de epidemias como el cólera, parecen haber sacudido algo las conciencias, no sólo por motivos sociales o ambientales, sino sobre todo por motivos económicos. Las inundaciones de 1993 en Norteamérica y Europa dejaron cuantiosas pérdidas, y la posible contaminación de algunos lagos del sur de Chile podría ser catastrófica no sólo desde el punto vista ecológico o emotivo, sino también porque traería aparejadas cuantiosas pérdidas económicas. (Vemos así que el tema no es privativo de países menos desarrollados.) Las consideraciones económicas, más que las ambientales, parece que finalmente inducirán a las esferas políticas y a las ejecutivas a pasar de las ideas a la acción.

Estas preocupaciones, sin embargo, aún no se han materializado en América Latina y el Caribe en la creación de organizaciones adecuadas para la gestión del agua. En general, el tema de la gestión de este recurso, tanto a nivel nacional como a nivel de cuencas, es bien acogido por muchas personas e instituciones, pero aún no llega a plasmarse en sistemas sólidos y estables - públicos, privados o mixtos-, salvo en contados casos y respecto de algunas cuencas donde por algún motivo se han realizado grandes inversiones en obras hidráulicas.

\section{VI}

\section{Recomendaciones para evitar fracasos en los procesos de gestión del agua y las cuencas}

Para abordar los variados factores que afectan la gestión ambiental a nivel de cuencas, de recursos naturales o sólo del agua, es necesario referirse al actual

\footnotetext{
${ }^{4}$ Así lo confirma la creación de las Corporaciones Autónomas Regionales (31) en Colombia, dependientes del Ministerio del Medio Ambiente (Ley 99 del 16 de diciembre de 1993). La función de estas corporaciones es la de ejecutar las políticas nacionales en materia de recursos naturales y medio ambiente.
}

debate sobre el papel del sector público y del sector privado. Es también necesario abordar el tema de las políticas hídricas y su vinculación con las políticas económicas y de participación democrática. Sólo entonces será factible determinar cuáles serían los sistemas de gestión más adecuados para el medio ambiente en general y las cuencas como parte de él.

El establecimiento de sistemas de gestión de aguas y de cuencas suele fracasar por la relativa lige- 
reza con que se presentan las propuestas de creación de las entidades pertinentes, sean ellas empresas públicas, corporaciones, comités, agencias u otras. En general se pretende darles un enfoque holístico: que sean económicamente eficientes, autosostenidas y competitivas; que tengan orientación social, que favorezcan la equidad y que sean ambientalmente responsables; que estén compuestas por sectores públicos y privados, que permitan la participación ciudadana y que sean de índole conciliadora y no autoritaria. Bien analizado, esto representa en esencia la aspiración a una superentidad que fomente el desarrollo sustentable. Gestar tal entidad se asemeja a crear genéticamente un animal que sea capaz de proporcionar carne y leche, $\mathrm{y}$ además ponga huevos $\mathrm{y}$ dé lana, y a la vez sea simpático y tolerante para tener cabida política, y no coma mucho para que mantenerlo no sea costoso.

La experiencia demuestra que se puede tender poco a poco a la formación de alguna entidad que por lo menos cumpla con algunas de las funciones básicas, como las de evitar, reducir o solucionar conflictos entre los usuarios del agua. Para hacerlo es necesario informarse de partida por lo menos sobre lo siguiente: i) las políticas referentes a los recursos hídricos y las políticas económicas; ii) las características propias de la gestión de los recursos hídricos y de las cuencas; iii) las características de los sistemas de gestión del agua y de los actores involucrados en ellos; iv) las formas más adecuadas de funcionamiento de una entidad pública o privada a cargo de la gestión del agua y los recursos naturales en una cuenca.

Según Irving Fox (1970), ${ }^{5}$ no hay consenso respecto de lo que debe entenderse por política de recursos hídricos. Sin embargo, el mismo autor indica que dichas políticas pueden caracterizarse por tres elementos que determinan cómo se manejan y utilizan estos recursos dentro de una determinada sociedad, a saber: las reglas básicas, los principios de organización y los procedimientos fundamentales.

Fox no define cada una de estas características; sólo pone ejemplos. Así, considera que las reglas básicas pueden referirse a temas como la asignación de derechos de agua y de prioridades de uso, si las hubiera, y otros semejantes. En cuanto a los principios de organización, un ejemplo podría ser el de estipular

\footnotetext{
${ }^{5}$ Este trabajo fue presentado por el Prof. Irving Fox, Director Asociado del Centro de Recursos Hídricos de la Universidad de Wisconsin, al panel de expertos de Naciones Unidas, Buenos Aires,
} 1970. en una política sobre el uso del agua que habrá un solo organismo a nivel de cada cuenca, formado conjuntamente por los usuarios y el Estado, con amplias atribuciones y autonomía para dirigir complejos programas de recursos hídricos con propósitos múltiples, regionalmente integrados; esto quiere decir que, como parte de la formulación de políticas, hay que considerar cómo se organizará el sistema de gestión para aplicarlas. Los procedimientos fundamentales se refieren a los medios, pasos o instancias para ejecutar las acciones: por ejemplo, estipular que para otorgar derechos de agua se debe probar previamente que existe el recurso, que no hay conflictos de uso con otros usuarios y que se utilizará con un determinado fin y dentro de un plazo establecido.

Para ser completa, por lo tanto, una política en materia de agua debería ser tanto de intención como de ejecución, independientemente de si merece calificarse de una u otra manera. Así, pues, la diferencia no estaría dada por el contenido, sino por la pròfundidad con que se detalla.

A las tres características básicas señaladas por Fox se agrega la necesidad de acompañar las declaraciones de política con el establecimiento de prioridades y la indicación, o por lo menos el esbozo, de los instrumentos de política para llevar a cabo la aplicación de las políticas formuladas.

Las declaraciones de política también pueden analizarse en función de la secuencia metodológica destinada a guiar procedimientos de gestión para el desarrollo sustentable (elaborada por A. Dourojeanni e incluida en CEPAL, 1993). Dicha secuencia es útil para orientar la formulación de políticas hídricas en una forma bastante más rigurosa que la habitual. En ella se expresa que para materializar acciones es preciso: i) determinar quiénes son los actores involucrados en el proceso de gestión; ii) precisar cuáles son los criterios de esos actores (políticas, principios, funciones y otros); iii) identificar qué problemas manifiestan en función de dichos criterios; iv) determinar cuáles son sus objetivos; v) delimitar los ámbitos dentro de los cuales se pretende alcanzar dichos objetivos; vi) precisar qué obstáculos existen para alcanzarlos; vii) proponer soluciones para superar tales obstáculos; viii) decidir las estrategias que se seguirán con miras a lograr las soluciones; ix) diseñar programas y proyectos para llevar a cabo las estrategias seleccionadas y evaluarlas, y x) llevar a cabo los programas y proyectos.

De conformidad con esta secuencia, la formulación de las políticas se efectúa primordialmente en el 
momento de tener que precisar los criterios de acción y los objetivos de los actores. Dichos criterios son en su mayoría declaraciones de intención. Las políticas de ejecución, en cambio, sólo pueden formularse al diseñar las soluciones y estrategias. Así, la formulación de políticas sobre el agua es un proceso que se ha de realizar paso a paso, en forma sistemática, a fin de no pasar por alto aspectos esenciales para aplicarlas con éxito.

En los países de la región pocas veces la formulación de políticas sobre el agua ha seguido un camino riguroso. En general se lleva a cabo a raíz de situaciones coyunturales, sin seguir un procedimiento establecido. Como se mencionó antes, las políticas de recursos hídricos de la región favorecieron en algunas oportunidades la formulación de planes, en otras la reformulación de una ley (no como culminación de un proceso de formulación de políticas en materia de agua sino como inicio de dicho proceso), en otras la creación de organizaciones y así sucesivamente. Sin embargo, preocupa que la gran mayoría de estas propuestas no se armonicen debidamente, pese a que los sistemas de organización, las leyes y los planes, por citar sólo algunos componentes de una política de ejecución, deben estar bien articulados.

En este contexto, se toman medidas parciales o cuyo objetivo es simplemente no contradecir un sistema económico, reforzar la aplicación de otras leyes (como la formulación de la ley general de aguas en el Perú, destinada a apoyar la ley de reforma agraria de 1969), paliar conflictos específicos y a veces circunstanciales entre usuarios, satisfacer a ciertos grupos electorales o conciliar algún proyecto de regionalización. En estas situaciones las políticas de aguas normalmente se formulan de manera subsidiaria e incompleta.

Aunque estas políticas deben apoyar a las políticas nacionales de desarrollo, cabe recordar que tanto los recursos hídricos como los procesos para su aprovechamiento tienen características propias que no pueden descuidarse sin caer en grandes contradicciones. Ya en 1970 Irving Fox advertía de los problemas que plantea pretender asociar la gestión del agua a una economía de libre mercado sin considerar las particularidades del caso. Las características propias del agua como recurso económico exigen forzosamente, si no la participación preponderante del Estado, como sostiene Fox, por lo menos una gestión conjunta del Estado y los usuarios para administrar su abastecimiento a nivel de una cuenca o sistema interconectado. (Véase el anexo 1).
En un sistema hídrico compartido por varios usuarios los servicios para satisfacer diferentes demandas pueden estar en manos privadas (agua potable, agricultura, hidroenergía y otros), pero la administración de la oferta de agua de la cuenca, incluidas todas las posibles formas de reaprovechamiento, debe estar a cargo de los usuarios y el Estado. Es la única manera de resolver los conflictos que pueden surgir, de aportar recursos para evitar problemas comunes como la contaminación y las inundaciones y de controlar externalidades (por ejemplo, el efecto de los aportes de ríos sobre las franjas costeras adyacentes a la desembocadura al mar). Este principio rige también en la organización de una comuna; si bien hay propiedades administradas por particulares, también debe existir una municipalidad que reglamente los ámbitos compartidos y evite que las propiedades privadas produzcan externalidades que afecten al resto de los habitantes.

La regionalización de algunos países ha originado profundas contradicciones entre las políticas de desarrollo y las políticas hídricas. Por ejemplo, en el Perú, en diversas oportunidades, debido a la regionalización aplicada por varios gobiernos, algunas autoridades de cuencas que dependían del gobierno central de pronto se encontraron supeditadas a dos y hasta tres autoridades regionales, porque la cuenca que administraban estaba dividida por los límites de varias regiones.

No es fácil precisar la forma y el grado en que las políticas de un país condicionan los procesos de gestión del agua. Dado que a menudo se desconocen las repercusiones que tienen las políticas vigentes en la gestión de los recursos hídricos, resulta difícil proponer fórmulas para mejorarlas. En otras palabras, si no se conoce cómo funciona la aplicación de las políticas que actualmente orientan el aprovechamiento del agua (causas y efectos), no es factible proponer mejoras con el fundamento necesario.

En muchos países no se cuenta con un catálogo de las leyes relativas a la gestión de los recursos hídricos y de las cuencas de captación. A veces tampoco hay registro de los usuarios por cuenca o sistema hídrico, ni un inventario de los estudios realizados en cada uno (Gómez y Dourojeanni, 1991). Es sencillo averiguar el contenido de las declaraciones de política, los organigramas y los reglamentos oficiales de funciones, pero es muy difícil saber en qué medida se llevan a la práctica. Gran cantidad de dependencias públicas no disponen de los recursos suficientes para cumplir con sus tareas. 
Por ahora, la mayoría de las políticas hídricas derivadas de los cambios en las políticas económicas no son más que declaraciones, es decir, políticas de intención. Sin embargo, cabe recordar que en muchas ocasiones, sin mayor profundidad de análisis, las políticas de intención se han transformado en leyes también de intención, lo que señala graves vacíos, sobre todo de instrumentos para llevarlas a la práctica. En varios casos no hay correlación entre el espíritu de la política, lo que aparece en la ley y lo que finalmente se logra con su aplicación.

El propósito de una entidad de gestión de cuencas se inscribe dentro de la función que poseen los Estados de tutelar el patrimonio natural de las naciones. Una entidad de este tipo puede ser de carácter público, privado o mixto, y puede adoptar diferentes formas jurídicas, pero es indispensable que opere de forma eficiente y eficaz, que sirva a sus usuarios al mismo tiempo que vele por el cumplimiento de las leyes nacionales sobre medio ambiente, y que realice su gestión con la participación de los actores involucrados, incluidos los municipios presentes en la cuenca.

Por el lado de las políticas económicas, la entidad de gestión de cuencas, para su financiamiento, usualmente se apropia de parte de la renta económica generada por el aprovechamiento de los recursos naturales de la región. Esta rentabilidad depende en parte de las características biológicas y fisicoquímicas que poseen los recursos naturales de aumentar su biomasa y mantenerse en la naturaleza a través de sus ciclos. Por ejemplo, la captación de hidroenergía es una apropiación de una riqueza natural.
La economía de los recursos naturales es todavía un tema incipiente que con el tiempo ayudará a tomar decisiones más acertadas en la definción de las fuentes de financiamiento para administrarlos. Por ejemplo, como señala el Banco Mundial (1993), el agua como bien adquiere diversas connotaciones según la capacidad de consumirla o no (subtractability) y de negar o no el acceso a más de un usuario (excludability): puede ser un bien público si es de libre acceso y no se consume si el uso de uno no impide el uso de otro, pero también puede ser un bien privado (por ejemplo, si está en la piscina de una casa). Es un bien de usufructo o de peaje (toll good), como el agua para la navegación; un bien de libre acceso (open access good) o un bien básico o de mérito (merit good), como el agua para beber.

Estas variaciones en las características del agua pueden servir para originar ingresos diferentes a una entidad encargada de coordinar sus utilización con fines múltiples. Para que así sea, la entidad debe disponer de registros que permitan medir los variados usos del agua, y evaluar su rentabilidad y los instrumentos para captar ingresos.

Desde el punto de vista teórico, el pago por el uso del recurso no debería alterar la racionalidad del cálculo capitalista de producción, ni distorsionar la formación de los precios de los bienes producidos en la cuenca. Considerando que el agua es un factor de producción, su uso debe verse reflejado en forma real en los costos de producción. En caso contrario se hace imposible su aprovechamiento a largo plazo, puesto que se tiende a su agotamiento y destrucción.

\section{VII}

\section{Recomendaciones para mejorar la formulación de políticas}

Hay algunas consideraciones básicas que se deben respetar si se desea formular políticas de gestión ambiental, de recursos naturales o sólo del agua que tengan un mínimo de coherencia. Si las políticas mismas son incoherentes también lo serán las entidades de gestión encargadas de llevarlas a la práctica.

En principio está claro que no cualquier declaración de principios califica necesariamente como una política, ni de intención (politics) ni de ejecución (policy). Para que lo sea debe contener reglas básicas, principios de organización y procedimientos fundamentales.

También es necesario que la declaración de cambios o la promulgación de nuevas políticas se haga considerando claramente las ventajas y desventajas de la aplicación de las políticas vigentes. De ninguna manera debe una nueva política empeorar la 
situación o socavar la eficacia de otra política recién promulgada.

Es importante además que las pruebas de las hipótesis implícitas en una política de intención se hagan aplicando métodos rigurosos, como el señalado en la secuencia de procedimientos de gestión para el desarrollo sustentable (CEPAL, 1993). Este requisito es esencial para pasar de políticas de intención a políticas de ejecución.

En materia ambiental, incluyendo el agua y otros recursos naturales, es necesario comprender e internalizar en la declaración de políticas hídricas las restricciones impuestas por el comportamiento y las necesidades del medio ambiente en general y del agua en particular. El sistema natural no modifica su comportamiento porque la sociedad humana decide cambiar de régimen o modalidad de gobierno y de sistema económico o comercial.

Sin lugar a dudas la declaración de políticas ambientales, y de políticas hídricas en particular, requiere que se consideren las políticas económicas vigentes y las funciones del sector privado y público. Ello es clave para determinar los instrumentos de política que se emplearán, así como el tipo de organización necesaria para aplicarlos.

En declaraciones de política ambiental, de gestión de recursos naturales o sólo del agua, es necesario precisar cuál será el sistema de organización que se aplicará y sobre todo cuáles serán los instrumentos económicos que se utilizarán para que funcione ese sistema, ya sea de carácter público, privado o mixto.

Una de las condiciones que exige el medio ambiente es la continuidad en las acciones: los sistemas de gestión ambiental deben perdurar más allá de la duración de un gobierno. Por ello es necesario que, como parte de la declaración de políticas, se acompañe un anteproyecto de ley y reglamentos que propicien la continuidad necesaria en el sistema de gestión, a fin de garantizar su financiamiento, efectividad, adaptación y legitimidad.

Para que una política ambiental tenga éxito debe ser comprendida por la población. En ese sentido, es importante que se indique quiénes participaron en la elaboración de la política y quiénes intervendrán en su posterior aplicación, así como la forma en que se tomaron las decisiones. Se deberá señalar el papel de cada actor y su participación relativa en el proceso de aplicación y acatamiento de instrumentos de política.

Es esencial que los objetivos económicos y am- bientales se compatibilicen, en momentos en que se tiende a aplicar una economía social y de mercado. Ambos tipos de objetivos pueden alcanzarse en la medida en que se establezca claramente el comportamiento de los sistemas económicos y ecológicos y se diseñen mecanismos de compatibilización.

Para ampliar este último punto con relación a un solo elemento, por ejemplo el agua, se exponen a continuación algunas ideas, sustentadas en un trabajo de Erhard-Cassegrain y Margat (1979).

Ante todo, en materia de recursos hídricos el razonamiento que se aplica para el rendimiento económico óptimo difiere del correspondiente al uso del agua: el rendimiento económico óptimo en un mercado se obtiene con la apertura a la competencia y la rivalidad; el rendimiento económico óptimo en el uso múltiple de los recursos hídricos se obtiene con un proceso objetivo, calculado y pensado, que cuente con la participación y el acuerdo de los actores que intervienen en él, incluido el Estado.

Lo anterior no significa que la aplicación de instrumentos económicos no sea útil y prioritaria para alcanzar los objetivos de uso óptimo del agua, sobre todo a nivel sectorial. Dichos instrumentos sirven para corregir y promover los esfuerzos del conjunto de usuarios, y sobre todo para obtener recursos destinados a combatir el efecto de fenómenos naturales adversos a todos ellos, como las inundaciones y las sequías, y a evaluar el agua de lluvia en los centros urbanos, disminuir la contaminación y evitar el uso inadecuado del territorio y sus recursos.

La demanda de un recurso natural como el agua tiene un doble efecto en el suministro. Por una parte, se extrae agua del medio (río, pozo) y se utiliza en diferentes formas; por otra, se la restituye al medio, en mayor o menor cantidad y calidad (al mismo río $u$ otro, o a la napa del subsuelo). Esta doble interacción no es común a todos los recursos que se comercializan en el mercado; así, pues, en la economía del agua no basta con considerar la eficiencia de extracción y uso, sino que también se debe considerar la eficiencia de la devolución.

La gestión del agua, como se destacó más atrás, es una gestión de conflictos entre usuarios que compiten por el mismo recurso, muchos de los cuales ni siquiera tienen una idea de cómo interactúan, perjudicándose o beneficiándose recíprocamente. La gestión del ambiente, y del agua en particular, debe ayudar a prevenir y evitar en lo posible dichos conflictos, estudiando las interrelaciones de los actores y sugiriendo negociaciones o transacciones ambientales entre ellos. 
En la formulación de políticas ambientales, sobre todo respecto del agua, muchas veces se separa arbitrariamente un sistema natural (ecosistema, cuenca) o un recurso como el agua, para su gestión por grupos de usuarios; por sectores responsables de su control; por usos de tipo consuntivo o no; por la fuente donde se capta el agua, sea ésta superficial o subterránea; por secciones o tramos de río, y otros criterios.

Se atomiza así arbitrariamente el sistema natural $\mathrm{y}$, en consecuencia, se realiza también en forma parcelada la gestion de un sistema naturalmente integrado. Según Erhard-Cassegrain y Margat (1979), no basta con racionalizar la entrega de la cantidad y calidad de agua a cada usuario si no se considera la forma en que ésta se va a restituir al medio (cantidad, calidad, lugar y tiempo). La restitución del recurso puede tener efectos tanto negativos como positivos: por ejemplo, una gran cantidad de agua de residuo doméstico reduce la concentración de elementos químicos procedentes de empresas que vierten residuos industriales líquidos, y sirve para su transporte.

El concepto de "economía del agua" no da resultado en el sentido material, es decir, de reducir al mínimo la cantidad de agua que se extrae del sistema o la que se usa en un proceso. Si ésta se reaprovecha en otros procesos, puede ser más rentable usar más agua en el primero. Por ejemplo, el uso de poca agua para riego en algunas partes redunda en un mayor costo y aumenta la concentración de sales en los suelos; sin embargo, si se puede captar, tratar y reaprovechar el agua posteriormente, podría ser más rentable utilizar más agua en el primer proceso.

No basta con asignar usos del agua por sectores (doméstico, agrícola, industrial) si no se consideran sus interacciones, ya que se produce un enfrentamiento entre cada tipo de demanda y el sistema de abastecimiento (agua subterránea para riego, agua de río para la industria, sección de río para las asociaciones de regantes, extracción de material del cauce para los municipios). Se separan así arbitrariamente las unidades físicas del sistema de abastecimiento en función de los diferentes usos, tal como se hace al dividir un río en secciones asignadas a diferentes grupos de usuarios, haciendo caso omiso de los efectos que tiene su interdependencia a lo largo del cauce. Con este enfoque las demandas de agua a veces se proyectan sólo por tipologías de oferta de recursos (demanda de aguas subterráneas para la ciudad, demanda de agua de río para la industria).

Las demandas se evalúan casi siempre según la cantidad y no la calidad, como si ambas fueran separables. Se olvida la acción recíproca de ambos factores en cada uno de los puntos de restitución de agua al sistema principal, y se olvida también que sacar agua o restituirla entraña cambios en su calidad y en la capacidad del medio de absorber ciertos contaminantes.

El valor del agua como insumo en un proceso productivo suele asignarse con independencia de la acción recíproca que existe entre la extracción, el uso y la restitución. Por ejemplo, cuando se fija un valor al agua no se asigna en forma preventiva un costo por los contaminantes que ésta va a verter a un cauce al restituir una cierta cantidad; tampoco se cobra por el uso del agua como medio de transporte y a veces como diluyente de residuos, además de su uso en algún proceso productivo.

Los costos de los efectos externos o externalidades negativas, como la contaminación producida por el uso del agua como medio de transporte de desechos, en definitiva son internalizados de acuerdo con el principio de que el contaminador paga. Sin embargo, habitualmente esta regla se aplica cuando han pasado varios años en que la industria ha dañado el medio, y los usuarios comienzan a quejarse. Para evitarlo se precisan acciones preventivas tanto en el cobro como en la aplicación de medidas de descontaminación. Entre el momento en que se contamina el agua y el momento en que se inicia su limpieza hay un período de inacción. Lo que sucede es que no se considera desde un principio que el usuario no sólo utilizará el agua en su proceso de producción, sino también como medio de transporte de sus residuos. Este uso podría calcularse y cobrarse al comienzo, inclusive estimando, por ejemplo, el costo alternativo para la fábrica de usar camiones para el transporte de sus desechos en lugar de usar el agua y los cauces.

El problema señalado se agrava exponencialmente porque los sistemas de gestión del abastecimiento de agua, que están igualmente fragmentados, no tienen mecanismos de coordinación. Unos se ocupan de la calidad del agua (como los ministerios de salud), otros de la extracción de áridos (como los municipios o los ministerios de minería), otros de las defensas fluviales, otros del agua subterránea, otros de otorgar derechos de uso, y así sucesivamente. La falta de coordinación deja muchos espacios vacíos en la gestión. Así se explica también por qué en muchos planes elaborados por estas entidades no se hace más que extrapolar las fragmentaciones expuestas. Por ejemplo, cada sector proyecta $s u$ demanda, pero no 
considera los efectos que tendrá en la cuenca ni cómo afectará al abastecimiento ni a las diversas demandas. De ahí que sean escasas las propuestas para reducir la demanda por iniciativa del propio sector que hace su plan en forma independiente. Todos reclaman el máximo, y si pueden se apropian preventivamente de la mayor cantidad posible de derechos de agua. Se entra en pugna por lo que se necesita hoy y por lo que acaso se necesitará mañana.

Así, pues, casi ninguna política sectorial busca modificar las tendencias de la demanda, por ejemplo, reasignando las industrias a lugares más apropiados; sólo se intenta satisfacerlas. No se contempla la opción de actuar a la vez sobre la oferta y la demanda, porque no existe un sistema de gestión integrado que dependa primordialmente de los propios usuarios y en virtud del cual se propongan medidas de beneficio tanto individual como colectivo para lograr economías de escala.

Cuando se carece de políticas de gestión integral se confunde el razonamiento técnico con el económico y éste, a su vez, con el financiero. En lugar de optar por un criterio de selección de objetivos que sirva para resolver las situaciones competitivas (recuérdese que la gestión del agua es una gestión de conflictos), se acaba por adoptar criterios útiles y sensatos para cada sector, pero insensatos para el conjunto, al punto de ocasionar mayores pérdidas a cada uno de ellos (mayores costos de regulación y captación de las aguas, de control de los fenómenos extremos y de descontaminación).
La maximización independiente del beneficio económico e hídrico de cada sector destruye el punto óptimo del conjunto. El resultado final es una pérdida social, económica y ambiental para todos. Se confunden las cosas si se entiende la economía del agua como una "economía material" del recurso. Parece racional economizar agua $\mathrm{y}$, por lo tanto, reducir el consumo; es también racional escoger la solución de abastecimiento de agua menos cara (en el sentido financiero); sin embargo, estos dos conceptos son contradictorios y a veces incompatibles si se toman como parte de un mismo objetivo.

Cuando no hay un sistema integrado de gestión también ocurre que los beneficios generados por un actor para otros usuarios del agua (como la reducción del efecto de las inundaciones o sequías gracias a la construcción de un embalse para una planta hidroeléctrica) no le son ni reconocidos ni pagados. En cambio los afectados reclaman y a veces sí cobran por los efectos negativos que puedan resultar. En consecuencia, la gestión del agua y la gestión económica deben considerarse desde arriba, en forma integral y no por partes. Si se analizan fragmentos se puede llegar a la errónea conclusión de que al optimizar materialmente cada uso del agua se optimiza la economía. En la práctica, la economía se encontrará en su mejor nivel cuando se analice el conjunto del sistema.

La selección de los instrumentos económicos de gestión más adecuados sólo puede hacerse teniendo en cuenta este carácter integral.

\section{VIII}

\section{Recomendaciones para mejorar las propuestas de creación de entidades de gestión de cuencas}

En principio, es posible corregir la forma inorgánica y poco fundamentada en que suelen plantearse las soluciones de tipo gerencial para mejorar el aprovechamiento de los recursos naturales. Dado que este artículo se refiere a las entidades a nivel de cuencas, se han listado los aspectos más relevantes con relación a estos sistemas de gestión.

Los principales factores que condicionan la estructura de una entidad de gestión de recursos naturales a nivel de cuenca son: i) la dimensión y las características ecológicas, climáticas, geomorfológicas y fisiográficas de la cuenca; ii) la organización y nivel de desarrollo de los municipios, el tipo de usuarios que predominan y sus poderes políticos y representatividad, así como su forma de participación en los gobiernos locales; iii) el grado de conocimiento de los elementos y recursos naturales de la cuenca, el tiempo de registros sobre el agua, el conocimiento del funcionamiento de los ecosistemas; iv) la organización vigente de la gestión en la cuenca por niveles de gestión (científico-ambiental, económico-productivo, técnico-normativo y político-social); v) los actores 
endógenos y exógenos que funcionan en la cuenca (número y características socioeconómicas); vi) la legalidad de la posesión o usufructo de los recursos naturales, propiedades y otros, así como la forma en que se agrupan actualmente los usuarios de la cuenca; vii) el grado de habilitación de la cuenca en materia de infraestructura vial, comunicaciones, transporte y otra infraestructura de servicios; viii) la posibilidad de participación de otras entidades en la gestión y los grados de coordinación y capacidad operativa (con el sistema judicial, policial, de investigación y capacitación, de laboratorios, de construcción y otros); ix) el nivel de actividad pública y privada en la cuenca (entidades existentes y funciones tanto de actores endógenos como exógenos), y x) la valorización económica de los recursos naturales presentes en la cuenca, así como la variedad de elementos naturales aún no valorizados económicamente (biodiversidad, paisajes, exclusividad).

Los aspectos en los que influyen los factores enumerados y que a la vez tipifican una entidad de cuencas son, entre otros:

i) las funciones de la entidad (coordinadora, fiscalizadora, planificadora, ejecutiva, administradora, concertadora, consultiva, controladora) y otras atribuciones vinculadas a su facultad de imponer o no decisiones para la solución de conflictos entre los usuarios de la cuenca; ii) las fuentes de financiamiento a las cuales tiene acceso la entidad de gestión de cuencas: impuestos territoriales, tarifas del agua, multas por contaminación, tesoro público, cuotas, fondos de proyectos, donaciones, venta de servicios, y otros; iii) la ubicación, dimensión y equipamiento de la entidad (oficinas, equipo de transporte, soporte computacional, sistemas de información); iv) el tipo y cantidad de personal y el organigrama interno de funcionamiento, que refleja la complejidad y tipo de conflictos de gestión presentes en la cuenca; v) el reglamento de operación y funciones, así como el presupuesto anual requerido para el funcionamiento de la entidad y para la inversión en proyectos; vi) el rango de autonomía frente al Estado y al comité directivo de la entidad (board) ante el cual el jefe de la entidad debe responder por el resultado de la gestión; vii) el grado y forma de participación de los actores involucrados en la gestión de la cuenca o afectados por dicha gestión (parlamento del agua, comité de cuenca u otro, de los cuales interesa conocer su composición y el peso relativo de su participación), y viii) la jerarquía de la entidad frente a la de otras entidades que actúan en la cuenca: su capa- cidad de coordinación y control, así como su poder de convocatoria.

La entidad a cargo de dirigir acciones integradas en una cuenca debe dimensionarse en función de los factores enunciados. Este listado no exhaustivo de aspectos que deben ser considerados pretende evitar que la formulación de propuestas para mejorar la labor de entidades públicas o mixtas encargadas de la gestión ambiental, los recursos naturales o el agua se base, como ha sido usual hasta ahora, en intuiciones, improvisaciones, emociones o acomodos políticos, más que en un trabajo riguroso de análisis.

Fórmulas usuales son, por ejemplo, proponer que se cree una comisión que estudie la situación y emita un diagnóstico; proponer un plan, de preferencia un "plan maestro"; modificar el nombre de las dependencias públicas competentes; unir o separar instituciones o partes de ellas; trasladar oficinas; cambiar el personal a cargo de las dependencias con cada cambio de autoridad superior; crear nuevos puestos o autoridades de confianza; encargar a una comisión de confianza que modifique la legislación vigente; entregar toda la responsabilidad de la gestión a los propios usuarios y renunciar al papel que le compete al Estado, o a la inversa; solicitar apoyo de algún organismo internacional, o ayuda bilateral por medio de un proyecto; solicitar alguna línea de crédito blando o donaciones bilaterales; convocar a grupos de expertos a talleres o seminarios para discutir el tema y cabildear; modificar los ámbitos de gestión ambiental, y descentralizar o centralizar el poder de gestión.

Cualquiera de estas medidas puede ser, en potencia, excelente; sin embargo, para que esto se concrete es necesario que se cumpla con una serie de requisitos, lo que pocas veces sucede. Entre esos requisitos figuran los siguientes:

i) que las recomendaciones estén debidamente basadas en estudios analíticos que tomen en cuenta la situación existente y todos los aspectos involucrados en un cambio de sistema de gestión; ii) que el momento en que se haga la propuesta sea políticamente adecuado y se determine de acuerdo con los intereses del país; iii) que los actores que participan en los sistemas de gestión del agua estén conscientes de la importancia y la necesidad de cooperar para que el manejo de este recurso sea integral; iv) que los propios usuarios sean capaces de financiar el proceso de gestión con sus aportes; v) que se asignen tareas específicas y que los acuerdos entre todos los que intervienen en los diversos niveles de la gestión del agua sean claros. 
Con el fin de contribuir a elaborar correctamente las propuestas de creación de entidades para la gestión de cuencas, se sugiere responder primero a las preguntas que se formulan en el anexo 2 , ya que este es el tipo de interrogantes que deben ser esclarecidas antes de proponer la creación de alguna entidad para la gestión de cuencas o de proponer soluciones para

\section{IX}

\section{Tareas futuras}

La estrategia más obvia frente a la falta de interés colectivo - sea por desconocimiento o por temor a sufrir las consecuencias de un sistema burocrático más- y a la carencia de recursos en la etapa inicial, es poner en marcha las autoridades de cuencas en forma escalonada.

Así, se puede partir por la creación de autoridades de aguas de la cuenca en lugar de autoridades de cuencas. Las autoridades de cuencas tienen funciones más amplias y difíciles de conciliar con las funciones de las autoridades de desarrollo regional. Las autoridades de aguas sólo se limitan a la administración del agua y al manejo de los recursos naturales asociados al agua de la cuenca y, por lo tanto, tienen menores conflictos de competencia con las autoridades nacionales y locales.

Las autoridades de agua de la cuenca deben tener, por lo tanto, a su cargo sólo la administración del uso múltiple del agua y el manejo de los recursos naturales de la cuenca con fínes de protección y conservación de la calidad del agua y de prevención y control de fenómenos extremos. En la práctica, deben manejar la oferta de los recursos hídricos de la cuenca. La creación específica de cada autoridad de aguas de las cuencas debe hacerse en forma progresiva, amparada por una ley general, de manera que los esfuerzos y recursos escasos se concentren para ayudar a la organización de los usuarios en algunas cuencas prioritarias, al tiempo que se adquiere experiencia en el tema.

Los usuarios del agua deben participar desde el inicio en la conformación de la autoridad de aguas de la cuenca a la que pertenecen. Esta autoridad debe estar conformada por los propios usuarios y los representantes del gobierno local y del Estado, $\mathrm{y}$ apoyada por un equipo técnico de planta permanente que le sirva de secretaría. El Estado puede aquellas entidades que han sido creadas pero son aún inoperantes. En América Latina y el Caribe hay muchas leyes estancadas por no haberse elaborado en forma completa y adecuada. La falta de claridad ha originado reacciones negativas antes de que nazca el sistema, sobre todo si las funciones y atribuciones no se han establecido con propiedad. comenzar las actividades organizando para las cuencas prioritarias una entidad de gestión del agua relativamente pequeña, con las características anteriores, pero que al iniciar sus trabajos disponga de una fuente fija de ingresos, basada tal vez en un impuesto territorial (por ejemplo, 50 centavos de dólar por año por cada 1000 dólares de valor de la propiedad).

Esta entidad, que puede llamarse agencia o corporación, debe solicitar que sean los propios usuarios quienes se organicen por cauces y canales, para registrarlos como usuarios y miembros potenciales del directorio o comité de aguas de la cuenca, así como para hacerse acreedores de beneficios de apoyo técnico y préstamos. La regularización de los usuarios, con indicación de volúmenes de agua, calidad, lugar, régimen y otros, puede ser hecha por consultores privados, técnicos y abogados, debidamente capacitados y reconocidos por el Estado. La regularización de los usos vigentes del agua, así como el conocimiento de los balances hídricos, deben ser condiciones previas para otorgar derechos de uso de agua.

Las decisiones de cobros especiales e inversiones las tomarán los representantes de los usuarios y los demás miembros del directorio de la cuenca. A medida que las mediciones de cantidad y calidad de agua sean más completas se podrá determinar mejor la forma de cobranza, el pago de costos por contaminación y la asignación de costos y beneficios de cada obra. Con los fondos que vaya recaudando, la autoridad de aguas de la cuenca, en coordinación con los sectores público y privado, debe ir equipando la cuenca con sistemas de medición de cantidad, calidad y frecuencia del agua, además de realizar estudios y asistir a los usuarios en asuntos técnicos $\mathrm{y}$ financieros. 
ANEXO 1

\section{Economías de empresa privada y gestión del agua}

En los países que confían en gran medida en la empresa privada para la producción y distribución de mercaderías y servicios se da por sentado, generalmente, que el mercado competitivo asigna los recursos y distribuye los servicios y mercaderías de acuerdo con el interés público general. Si esto es así, ¿por qué tienen estos países necesidad de que el gobierno establezca una política de recursos hídricos? La respuesta es que, aun en los casos en que el mercado privado funciona razonablemente bien como mecanismo de asignación de recursos, hay una cantidad de razones que hacen necesaria la acción del gobierno para asegurar una inversión apropiada en recursos hídricos y para conseguir una adecuada producción y distribución de los suministros y servicios hídricos.

Esto se debe a que es más dificil determinar los derechos de propiedad sobre el agua que sobre la mayoria de los otros recursos. La determinación precisa de los derechos de propiedad es esencial para el buen funcionamiento de las instituciones económicas dentro de una empresa privada. Como el agua fluye de un lugar a otro (los abogados se refieren a ella como a un recurso "fugitivo"), y como este fluir varía de acuerdo con las diferentes condiciones hidrológicas, la determinación de los derechos de propiedad sobre el agua ha planteado problemas difíciles. En aquellos lugares donde el agua y su utilización están intensamente desarrolladas, ha sido necesario establecer una legislación precisa que determine los derechos al uso del agua. Para que esta legislación esté al servicio del interés público se habrá de tomar en cuenta los objetivos nacionales y escalas de valores y la legislación tendrá que elaborarse para servir a estos principios y valores. En resumen: la legislación relativa a la distribución hídrica debe estar al servicio de los objetivos de la política nacional.

Otra razón por la cual la economía privada no llega a resultados óptimos en el libre juego de las fuerzas de mercado en el campo de la administración de los recursos hídricos es la existencia de importantes economías de escala en el aprovechamiento hídrico, que hacen que sea antieconómica la competencia entre varias unidades dedicadas a la producción y distribución de agua.

Por ejemplo, no puede haber medios que compitan en el suministro de agua para uso familiar o para propósitos agrícolas. Es antieconómico que haya canales de navegación, o aun sistemas de energía eléctrica, que sean competitivos en una misma region. Sin embargo, la competencia es esencial en una economía de empresa privada no regulada si se desẹa obtener resultados razonablemente buenos.

Un tercer factor que inhibe el funcionamiento de un sistema no regulado de empresa privada en el campo de los recursos hídricos es la importancia de lo que podría llamarse efectos "externos" del uso del agua. Un depósito situado en un punto de la cuenca de un río tiene influencia sobre los caudales y, por lo tanto, afecta los beneficios y costos de las localidades ubicadas corriente abajo. Para alcanzar los beneficios máximos de la energía hidroeléctrica, los depósitos de reserva que se encuentran en la cuenca de un río deben proyectarse y administrarse de manera tal que se tengan en cuenta estas interrelaciones. Cuando se usa una corriente de agua para deshacerse de la basura, estos efectos "externos" (contaminación) pueden ser de carácter perjudicial. En los casos en que varias unidades de empresa privada estén comprometidas en el aprovechamiento y la administración de un sistema fluvial, estos efectos "externos", a la vez positivos y negativos, pueden no ser tomados en cuenta, a menos que la acción gubernamental así lo requiera; si no lo son, no se alcanzarán resultados eficientes.

Hay aún otra razón por la cual un sistema de empresa privada no está en condiciones de obtener resultados optimos en ausencia de una acción gubernamental y es que algunos de los servicios derivados del aprovechamiento hidráulico no pueden ser divididos en unidades, a los efectos de la compra y venta en un mercado competitivo. Por ejemplo, cuando se levanta una construcción para controlar los efectos de las crecientes, esta obra protegerá a todos los dueños de propiedades a lo largo de un trecho del río en forma tal que no se dará a los propietarios la opción para decidir si cada uno compra o no esta protección. Del mismo modo, si se trata de valores de recreación, estos valores beneficiarán al público en general y no se pueden comprar y vender en el mercado.

El resultado neto de las múltiples características físicas y económicas de los recursos hídricos es que, aun en una economía de empresa privada, se requiere en gran medida la intervención pública, si se desea que el aprovechamiento y manejo hídricos estén al servicio de los objetivos y escalas de valores de la sociedad. La intervención del gobierno, por su parte, debe estar regida por una serie de políticas y reglas relacionadas, por un lado, con los objetivos sociales y, por el otro, con las características físicas y económicas de los recursos hídricos.

Fuente: Irving K. Fox, Problemas de política hídrica, Recursos hídricos, vol. 1, N 3, Buenos Aires, 1970. 


\section{Preguntas para diseñar las propuestas de creación de entidades para la gestión de cuencas hidrográficas}

- ¿Qué tipo de entidad se está proponiendo según la clasificación presentada en el cuadro 1 ? ¿Quién propone la creación de esta entidad y por qué?

- ¿Qué evolución han tenido otras entidades a nivel de cuencas dentro del país? ¿Cuáles han sido esas entidades y qué experiencias han tenido?

- ¿Qué pasaría si no se estableciera ningún sistema de coordinación de acciones en la cuenca?

- ¿Es o no es necesario llevar a cabo algún tipo de coordinación de las acciones que se realizan en una cuenca? ¿Cuáles acciones deberían ser coordinadas? ¿Qué actores están interesados?

- ¿Quiénes están a cargo actualmente de ejecutar en la cuenca acciones que deberían ser coordinadas?

- ¿Qué ganarían los actores participantes si coordinaran algunas acciones relevanes en la cuenca, como por ejemplo el uso múltiple del agua? ¿Cambiaría la situación actual? ¿Cómo?

- ¿Qué perderían los actores en el caso de que se montara un sistema para coordinar acciones relevantes en la cuenca?

- ¿Cuáles son los principales obstáculos que impiden actualmente hacer operativo un sistema de gestión coordinada de las acciones en la cuenca?

- ¿Qué tipo de argumentos a favor y en contra sostie- nen los diferentes actores para crear o no crear un sistema de coordinación de acciones en la cuenca?

- ¿Qué tipo de sistema es necesario crear para coordinar las acciones? ¿Una autoridad (corporación, agencia), una oficina o secretaría técnica? ¿Una simple comisión de coordinación?

- ¿Qué funciones y atribuciones tendría tal sistema o entidad? ¿A qué se dedicaría? ¿Cómo estarían representados los usuarios de la cuenca? ¿Qué fuerza legal tendrían en las decisiones los diferentes actores?

- ¿Qué entidades existen actualmente, dentro o fuera de la cuenca, que puedan apoyar las tareas de la entidad que se propone (por ejemplo, el sistema judicial, el sistema policial y los servicios de extensión y capacitación rural)?

- ¿Qué sistema de financiamiento existiría para la entidad que se proponga? ¿Quiénes van a aportar los recursos para la coordinación, cuándo, cuánto y cómo? ¿Qué sistema de cobranza se va a tener? ¿A qué se destinarían los fondos captados?

- ¿Cómo se organizaría esta entidad? ¿Con qué sistema de información podría operar?

- ¿Qué tipo de atribuciones legales tendría para poner en vigencia los acuerdos que se tomen para intervenir coordinadamente en la cuenca? ¿Podría proponer planes para la ejecución de acciones coordinadas? ¿Cómo garantizaría su aplicación?

\section{Bibliografía}

Acero Suárez, José (1993): Proyecto para la recuperación y manejo de la cuenca hidrográfica del río Chicamocha, Colombia hídrica, año 6, $\mathrm{N}^{\circ} 8$, Bogotá, Asociación Colombiana de Ciencias Hídricas, mayo.

Banco Mundial (1993): Water Resources Management, World Bank Policy Paper, Appendix A, "Market failures and public policy in water management", Banco Mundial.

Cano, Guillermo y Joaquín López (1976): Las cuencas hídricas como unidades óptimas para la clasificación y administración de los recursos hídricos: participación de los usarios en tales actividades, trabajo preparado para el cincuentenario de la creación de las confederaciones de cuencas en España, Zaragoza, España.

CEPAL (Comisión Económica para América Latina y el Caribe)
(1993): Procedimientos de gestión para el desarrollo sustentable (Aplicables a municipios, microrregiones y cuencas), LC/G. 1769, Santiago de Chile, 31 de agosto.

Erhard-Cassegrain, A. y J.F. Margat (cons.) (1979): Schizophrenic Rationality and Water Resources Utilization, Water/ Serm. 6/R. 20, Ginebra, Naciones Unidas, Comisión Económica para Europa (CEPE).

Fox, Irving K. (1970): Problemas de política hídrica, Recursos hídricos, vol. 1, $\mathrm{N}^{\circ} 3$, Buenos Aires.

Gómez, Juan y Axel Dourojeanni (1991): Sistema de gestión del agua en la cuenca del río Mapocho, Anales del seminario sobre gestión de los recursos naturales renovables en la cuenca del Itata, Chillán, Chile, Universidad de Concepción/Gobernación Provincial de Ñuble. 